MIT-LNS-02-297

MIT-CTP-3249

hep-th/0202139

\title{
Anomalous reparametrizations and butterfly states in string field theory
}

\author{
Martin Schnabl ${ }^{1}$ \\ Center for Theoretical Physics, Massachusetts Institute of Technology, \\ Cambridge, MA 02139, USA
}

\begin{abstract}
The reparametrization symmetries of Witten's vertex in ordinary or vacuum string field theories can be used to extract useful information about classical solutions of the equations of motion corresponding to D-branes. It follows, that the vacuum string field theory in general has to be regularized. For the regularization recently considered by Gaiotto et al., we show that the identities we derive, are so constraining, that among all surface states they uniquely select the simplest butterfly projector discovered numerically by these authors. The reparametrization symmetries are also used to give a simple proof that the butterfly states and their generalizations are indeed projectors.
\end{abstract}

\section{Introduction}

The open string field theory [1] has so far resisted all the attempts to find analytically its solutions corresponding to the true closed string vacuum or lower dimensional Dbranes. On the other hand the solutions are known numerically $[2,3]$ and provide a good evidence for the Sen's tachyon conjectures [4]. The vacuum string field theory (VSFT) $[5,6]$, designed to describe the physics around the true tachyon vacuum has been conjectured to have purely ghost kinetic term, which then allows for some exact

\footnotetext{
${ }^{1}$ E-mail: schnabl@lns.mit.edu
} 
calculations. Canonical choice of a ghost kinetic term has been recently proposed by Gaiotto, Rastelli, Sen and Zwiebach [7] based on some earlier results of Hata and Kawano [8]. It has been further discussed by Okuyama [9].

Generic feature of pure ghost kinetic terms in VSFT is that they allow for simple construction of solutions in terms of projectors in the matter part of the string field algebra. It has been proved that such solutions indeed have the right ratios of tensions to be interpreted as D-branes $[10,11]$. To determine however the absolute normalization turned out to be quite problematic. As we will show, on general grounds one should expect some sort of divergences in the normalization of the action. To deal with the problem, the authors of [7] added a small term to the kinetic operator proportional to $c_{0} L_{0}$. With this regularization they observed that the numerical solution in the limit of removed regulator approaches a new type of a projector, called butterfly projector. The poetic name comes from the shape of the surface which represents these kind of states in the conformal field theory. Some properties of these states were also reported in [12]. For other very recent development in the VSFT see [27, 28, 30, 29, 31, 32].

In this paper we would like to get more insight into the problem of classical solutions and butterfly states in VSFT by an extension of the analysis of our earlier work [13]. The basic idea is to study implications of anomalous symmetries of the Witten's vertex for classical solutions of the action. These techniques turn out to be remarkably useful in this new context. They show that in the vacuum string field theory one inevitably encounters some sort of singularities. For the regularized version of the theory we obtain infinite set of nontrivial identities which, as we show, are fulfilled for the simplest butterfly state. The identities are so constraining, that among all well defined surface states they uniquely pick up one particular state, which even happens to be a projector. It would be rather interesting if one could derive more identities which would then determine the projector uniquely in the the whole Hilbert space, not necessarily in the subspace of surface states.

It turns out to be possible to express any well defined surface state as a reparametrization of the vacuum in the form

$$
\langle 0| U_{f}=\langle 0| e^{K}
$$

which greatly simplifies calculations of some star products. Here $K$ stands for some linear combination of the generators $K_{n}=L_{n}-(-1)^{n} L_{-n}$, which are known to act 
as exterior derivatives in the string field algebra. This fact has been shown in a recent paper [14] for wedge states. It allowed to determine the star products of wedge states purely algebraically, without thinking much about gluing surfaces and various coordinate systems.

Particularly interesting class of states is the one of the form

$$
\lim _{\alpha \rightarrow \pm \infty}\langle 0| e^{\alpha K}
$$

If the limit exists, then it gives states which are invariant under the reparametrization $K$, i.e. they are annihilated by $K$. These states are natural candidates for being projectors. For example the simplest butterfly surface state

$$
\langle 0| e^{-\frac{1}{2} L_{2}}=\lim _{\alpha \rightarrow-\infty}\langle 0| e^{\alpha K_{2}}
$$

is a projector. On the other hand the opposite limit

$$
\langle 0| e^{+\frac{1}{2} L_{2}}=\lim _{\alpha \rightarrow+\infty}\langle 0| e^{\alpha K_{2}} .
$$

is not a projector, we call it a 'false butterfly' state. These kind of states $(3,4)$ can be naturally generalized by replacing $K_{2}$ with $K_{2 k}$.

The paper is organized as follows: In section 2 we study the consequences of the anomalous symmetries of the Witten's three vertex for the classical solutions of the ordinary, vacuum and regulated vacuum string field theories. We derive an infinite set of identities, which as we show are obeyed by the simplest butterfly state. We then show in section 3, that the butterfly states and their generalization are indeed projectors. We manage also to calculate the star product $e^{s L_{-2}}|0\rangle * e^{s L_{-2}}|0\rangle$ for arbitrary $s \in\left[-\frac{1}{2}, \frac{1}{2}\right]$. In section 4 we prove the uniqueness of the solution of the set of identities among the surface states. As a corollary we obtain a formula (1). Appendices contain some useful formulas for the exponentials of the Virasoro operators, conservation laws for butterfly states and some numerical results for illustrative purposes.

Related work on butterfly states with some small overlaps with our work has been done independently by Gaiotto, Rastelli, Sen and Zwiebach and should appear simultaneously in [33]. 


\section{Constraints from anomalous symmetries}

To start with, we shall recall first some identities for the tachyon condensate found in our earlier work [13].

\subsection{Constraints in the Witten's string field theory}

The action of Witten's string field theory [1] takes a nice form of noncommutative Chern-Simons action

$$
S[\Phi]=-\frac{1}{\alpha^{\prime} g_{o}^{2}}\left(\frac{1}{2}\langle\Phi, Q \Phi\rangle+\frac{1}{3}\langle\Phi, \Phi * \Phi\rangle\right),
$$

where $Q$ is the usual string BRST operator. The star multiplication is given by a three vertex $\langle V|[15,16]$ which satisfies various identities $[15,17,18]$. For our purposes the most important are

$$
\begin{aligned}
\langle V| \sum_{i=1}^{3}\left(L_{-n}^{X(i)}-L_{n}^{X(i)}\right) & =3 k_{n}^{x}\langle V|, \\
\langle V| \sum_{i=1}^{3}\left(L_{-n}^{g h(i)}-L_{n}^{g h(i)}\right) & =-3 k_{n}^{x}\langle V|, \\
\langle V| \sum_{i=1}^{3}\left(J_{-n}^{(i)}+J_{n}^{(i)}\right) & =3\left(h_{n}^{g h}+3 \delta_{n, 0}\right)\langle V|,
\end{aligned}
$$

valid for $n$ even and $L_{n}^{X}$ and $L_{n}^{g h}$ denote matter or ghost Virasoro operators and $J_{n}$ are ghost current generators. The constants $k_{n}^{x}$ and $h_{n}^{g h}$ for $n$ even are given explicitly by

$$
\begin{aligned}
k_{n}^{x} & =\frac{13 \cdot 5}{27} \cdot \frac{n}{2}(-1)^{\frac{n}{2}}, \\
h_{n}^{g h} & =-(-1)^{\frac{n}{2}} .
\end{aligned}
$$

Taking the variation of the action (5) under the infinitesimal variations of the string field $\Phi$

$$
\begin{aligned}
\delta \Phi & =\left(L_{-n}^{X}-L_{n}^{X}-k_{n}^{x}\right) \Phi \\
\delta \Phi & =\left(J_{-n}+J_{n}-h_{n}^{g h}-3 \delta_{n, 0}\right) \Phi
\end{aligned}
$$


we see that they leave the cubic term in the action invariant. On the other hand we know that the total action should also be invariant, as long as $\Phi$ satisfies equations of motion. Combining these two facts we get from the kinetic term

$$
\begin{aligned}
\left\langle\Phi\left|\left[Q, L_{n}^{X}\right]\right| \Phi\right\rangle & =-k_{n}^{x}\langle\Phi|Q| \Phi\rangle \\
\left\langle\Phi\left|\left[Q, J_{n}\right]\right| \Phi\right\rangle & =h_{n}^{g h}\langle\Phi|Q| \Phi\rangle .
\end{aligned}
$$

These identities in the Siegel gauge simplify to

$$
\begin{aligned}
\left\langle\Phi\left|c_{0} L_{n}^{X}\right| \Phi\right\rangle & =\frac{1}{n} k_{n}^{x}\left\langle\Phi\left|c_{0} L_{0}^{t o t}\right| \Phi\right\rangle, \\
\left\langle\Phi\left|c_{0}\left(n J_{n}+L_{n}^{t o t}\right)\right| \Phi\right\rangle & =-h_{n}^{g h}\left\langle\Phi\left|c_{0} L_{0}^{t o t}\right| \Phi\right\rangle,
\end{aligned}
$$

where $L_{n}^{\text {tot }}=L_{n}^{X}+L_{n}^{g h}$ is the total Virasoro generator. They can be further rewritten as

$$
\begin{aligned}
\left\langle\Phi\left|c_{0} L_{n}^{X}\right| \Phi\right\rangle & =+\frac{65}{54}(-1)^{\frac{n}{2}} K, \\
\left\langle\Phi\left|c_{0} L_{n}^{\prime g h}\right| \Phi\right\rangle & =-\frac{11}{54}(-1)^{\frac{n}{2}} K,
\end{aligned}
$$

where $K=\left\langle\Phi\left|c_{0} L_{0}^{\text {tot }}\right| \Phi\right\rangle$ and $L_{n}^{\prime g h}=L_{n}^{g h}+n J_{n}+\delta_{n, 0}$ are Virasoro operators of the twisted ghost conformal field theory [7] with central charge $c=-2$. Note that the operators $L_{n}^{\prime g h}$ are singlets under the $S U(1,1)$ symmetry in the Siegel gauge, discussed in $[19]$.

\subsection{Constraints in the vacuum string field theory}

The basic idea of the vacuum string field theory (VSFT) is that when the Witten's string field theory is expanded around the nonperturbative vacuum, its action can be again written in the form

$$
S[\Psi]=-\kappa_{0}\left(\frac{1}{2}\langle\Psi, \mathcal{Q} \Psi\rangle+\frac{1}{3}\langle\Psi, \Psi * \Psi\rangle\right),
$$

where $\Psi$ is the fluctuation around the new vacuum after suitable reparametrization and $\mathcal{Q}$ is some new BRST-like operator which is nilpotent, acts as a derivation of the string field algebra and is conjectured to be purely ghost. Recently it has been argued 
$[7,9]$, that there are certain singular reparametrizations which lead naturally to one particular kinetic operator

$$
\mathcal{Q}=c_{0}+\sum_{k=1}^{\infty}(-1)^{k}\left(c_{2 k}+c_{-2 k}\right) .
$$

The major advantage of VSFT with the purely ghost kinetic operator $\mathcal{Q}$ is that it allows for constructing D-branes in terms of projectors of the algebra. They have been shown exactly, using the methods of boundary conformal field theory and Fock space oscillators, to have the right ratios of tensions $[10,11]$. What is still missing, is to show that there is a solution corresponding to the original unstable D25-brane with the right tension. We will see that the analysis of the constraints analogous to those discussed in the previous subsection can shed some light on the nature of this D25-brane solution.

Let $\Psi_{0}$ be a stationary point of the action (12), i.e. a solution of the equations of motion $\mathcal{Q} \Psi_{0}+\Psi_{0} * \Psi_{0}=0$. Then the identities read

$$
\begin{aligned}
\left\langle\Psi_{0}\left|\left[\mathcal{Q}, L_{n}^{X}\right]\right| \Psi_{0}\right\rangle & =-k_{n}^{x}\left\langle\Psi_{0}|\mathcal{Q}| \Psi_{0}\right\rangle, \\
\left\langle\Psi_{0}\left|\left[\mathcal{Q}, L_{n}^{g h}\right]\right| \Psi_{0}\right\rangle & =k_{n}^{x}\left\langle\Psi_{0}|\mathcal{Q}| \Psi_{0}\right\rangle, \\
\left\langle\Psi_{0}\left|\left[\mathcal{Q}, J_{n}\right]\right| \Psi_{0}\right\rangle & =h_{n}^{g h}\left\langle\Psi_{0}|\mathcal{Q}| \Psi_{0}\right\rangle .
\end{aligned}
$$

For a generic $\mathcal{Q}$ which is purely ghost, the first equation implies that

$$
\left\langle\Psi_{0}|\mathcal{Q}| \Psi_{0}\right\rangle=0 .
$$

It seems therefore to show that the tension of the D25-brane

$$
\frac{\kappa_{0}}{6}\left\langle\Psi_{0}|\mathcal{Q}| \Psi_{0}\right\rangle
$$

and of any other soliton is zero. The same conclusion follows for the particular choice (13) also from a combination of the second and third identity of (14) in the Siegel gauge.

So, does this mean that the VSFT cannot describe finite tension D25-branes? No, the argument merely shows that for the soliton solutions things become singular and one must be very careful. In the scenario advocated in [7] for the emergence of purely ghost kinetic operator in VSFT, the constant $\kappa_{0}$ in front of the action becomes naturally 
singular. When the action is carefully regularized we may hope to get the right finite answer.

One might be curious whether the above argument definitely rules out the possibility that one day we find completely regular VSFT action with purely ghost kinetic term and with finite action solutions. As a possible example of what could happen, the following toy version of VSFT has been proposed by [7, 20]. Suppose we start with a string field of ghost number zero and consider an operator $Y(z)=\frac{1}{2} c \partial c \partial^{2} c(z)$, which is a dimension zero primary of ghost number three. Take an action of the form

$$
S=\beta\left[\frac{1}{2}\langle\Phi|(Y(i)+Y(-i))| \Phi\rangle+\frac{1}{3}\langle\Phi|(Y(i)+Y(-i))| \Phi * \Phi\rangle\right] .
$$

From the argument in [7] it follows that for projectors which are surface states in the total Virasoro algebra (the sliver for example), the action is finite and equal to $\frac{1}{3} \beta$. So where does the argument breaks down? It is quite simple to find few places in the case of the butterfly projector

$$
|B\rangle=e^{-\frac{1}{2} L_{-2}}|0\rangle,
$$

discovered in [7] and discussed in more detail in section 3 and in $[12,33]$. Easy check shows, that for example the conservation laws (6) break down. Indeed, for $n=2$

$$
\begin{aligned}
& \left\langle V\left|\sum_{j=1}^{3}\left(L_{-2}^{X(j)}-L_{2}^{X(j)}\right)(Y(i)+Y(-i))\right| B^{(1)}\right\rangle \otimes\left|B^{(2)}\right\rangle \otimes\left|B^{(3)}\right\rangle=\frac{3 c}{4} \\
& \neq 3 k_{2}^{x}\left\langle V|(Y(i)+Y(-i))| B^{(1)}\right\rangle \otimes\left|B^{(2)}\right\rangle \otimes\left|B^{(3)}\right\rangle=-\frac{5 c}{18}
\end{aligned}
$$

where $c=26$ is the central charge in the matter sector. In section 3 we will see that some things are actually ill defined, for instance the inner product $\langle B|(Y(i)+$ $\left.Y(-i)) L_{-2}^{X} B\right\rangle$ and even the star product $|B\rangle * L_{-2}^{X}|B\rangle$ itself.

To conclude, our argument rules out possibility of finite action solutions which are sufficiently regular, it allows however for less well behaved solutions as are the projectors.

\subsection{Constraints in the regularized VSFT}

In [7] the following regularized action of the VSFT in the Siegel gauge has been proposed

$$
S_{a}[\Psi]=-\kappa_{0}(a)\left(\frac{1}{2}\left\langle\Psi, c_{0}\left(1+\frac{1}{a} L_{0}^{t o t}\right) \Psi\right\rangle+\frac{1}{3}\langle\Psi, \Psi * \Psi\rangle\right) .
$$


The original gauge fixed VSFT is recovered in the limit $a \rightarrow \infty$. It is perhaps curious that the other limit $a \rightarrow 0$ corresponds to the ordinary Witten's string field theory. One might therefore hope that both actions share some features, and that is indeed true. Let us write the constraints on the solutions to the equation of motion. Since we are in the Siegel gauge, we may write only identities based on variations $\delta \Psi$, which preserve the gauge

$$
\begin{aligned}
\left\langle\Psi_{0}\left|c_{0} L_{n}^{X}\right| \Psi_{0}\right\rangle & =\frac{65}{54}(-1)^{\frac{n}{2}} a K, \\
\left\langle\Psi_{0}\left|c_{0}\left(n J_{n}+L_{n}^{g h}\right)\right| \Psi_{0}\right\rangle & =-\frac{11}{54}(-1)^{\frac{n}{2}} a K,
\end{aligned}
$$

where

$$
K=\left\langle\Psi_{0}\left|c_{0}\left(1+\frac{1}{a} L_{0}\right)\right| \Psi_{0}\right\rangle
$$

It was observed in [7] that in the limit $a \rightarrow \infty$ the numerical solution of the equations of motion representing the D25-brane seems to approach the following state

$$
\left|\Psi_{0}\right\rangle=\mathcal{N} e^{-\frac{1}{2}\left(L_{-2}^{X}+L_{-2}^{\prime g h}\right)} c_{1}|0\rangle .
$$

We will now show that this solution is compatible with the infinite set of identities (21) even for finite $a$. An immediate question is then, whether the solution (23) is a unique solution of this set of identities. We will prove in section 4 , that it is indeed unique among the surface states in the deformed CFT. It is most likely not unique among non-surface states, since for example in the limit $a \rightarrow 0$ corresponding to ordinary string field theory, we have another solution. To deal with non-surface states, one would almost certainly need more identities, which would differ from (21) by taking expectation values of products of more operators.

To check the identities we have to first regularize the solution (23)

$$
\left|\Psi_{0}^{s, \tilde{s}}\right\rangle=\mathcal{N} e^{s L_{-2}^{X}+\tilde{s} L_{-2}^{g h}} c_{1}|0\rangle
$$

keeping $|s|$ and $|\tilde{s}|<\frac{1}{2}$, and let $s$ and $\tilde{s}$ approach $-\frac{1}{2}$. Notice, that the regularized solution is not necessarily a solution of the regularized action. We are taking fixed finite $a$ and want to show that the identities are obeyed in the limit $s, \tilde{s} \rightarrow-\frac{1}{2}$.

Let us focus first on the identity

$$
\frac{\left\langle\Psi_{0}\left|c_{0} L_{2}^{X}\right| \Psi_{0}\right\rangle}{\left\langle\Psi_{0}\left|c_{0} L_{2}^{\prime g h}\right| \Psi_{0}\right\rangle}=-\frac{65}{11},
$$


which follows from (21). By a straightforward calculation, whose details are given in Appendices B and $\mathrm{C}$, we see that we have to require that in our limit

$$
\frac{1-4 \tilde{s}^{2}}{1-4 s^{2}}=\frac{5}{11}
$$

is kept fixed. Remarkably, making this single adjustment suffices to show that all other identities (21) are satisfied. Using the formulas collected in Appendix C we get

$$
a K=\left\langle\Psi_{0}\left|\left(a c_{0}+c_{0} L_{0}^{t o t}\right)\right| \Psi_{0}\right\rangle \approx \frac{27}{5}\left(\frac{5}{11}\right)^{\frac{1}{4}} \mathcal{N}^{2} \frac{1}{\left(1-4 s^{2}\right)^{3}}
$$

Here it is important, that we take first the limit $s, \tilde{s} \rightarrow-\frac{1}{2}$ for fixed $a$, and only at the end when we get $a$ independent ratios we may send $a \rightarrow \infty$, if we wish so. We have also

$$
\begin{aligned}
\left\langle\Psi_{0}\left|c_{0} L_{2 k}^{X}\right| \Psi_{0}\right\rangle & \approx(-1)^{k} \frac{26}{4}\left(\frac{5}{11}\right)^{\frac{1}{4}} \mathcal{N}^{2} \frac{1}{\left(1-4 s^{2}\right)^{3}} \\
\left\langle\Psi_{0}\left|c_{0} L_{2 k}^{\prime g h}\right| \Psi_{0}\right\rangle & \approx-(-1)^{k} \frac{11}{10}\left(\frac{5}{11}\right)^{\frac{1}{4}} \mathcal{N}^{2} \frac{1}{\left(1-4 s^{2}\right)^{3}}
\end{aligned}
$$

The identities (21) now immediately follow from (27) and (28).

Let us make one more remark about the logic here. We have shown that with a finite regularization $a$ of the VSFT action, the butterfly state exactly solves the infinite set of identities. In order to prove that, we had to regularize the state as we did. We have not shown, that it exactly obeys the equations of motion of the regularized theory. We will see in section 3.2 however, that the regularized butterfly approximately solves the equations of motion of the regularized VSFT, provided we identify $s=\tilde{s}$ and let it depend on $a$ in a definite way.

\section{Butterfly states}

Butterfly states were introduced first in [7, 12] as surface states associated to the conformal mappings ${ }^{2}$

$$
\begin{aligned}
f_{r}(z) & =\sin \left(\frac{2}{r} \arctan z\right) \\
& =\frac{z}{\sqrt{1+z^{2}}} \circ \tan \left(\frac{2}{r} \arctan z\right)
\end{aligned}
$$

\footnotetext{
${ }^{2}$ Our parameter $r$ is related to the parameter $\alpha$ of Gaiotto et al. [7] by $r=\frac{2}{\alpha}$.
} 
Various descriptions of the surface states have been developed recently in [10]. Let us review some definitions and properties we will need later. For a conformal field theory (CFT) defined on upper half plane, take a map $f(z)$ which maps real axis to real axis and the upper unit half disk one to one in the upper half plane. The surface state associated to the map $f$ is defined as a linear functional on the Hilbert space, which is just a bra vector, satisfying

$$
\langle f \mid \phi\rangle=\left\langle 0\left|U_{f}\right| \phi\right\rangle=\langle f \circ \phi(0)\rangle, \quad \forall \phi .
$$

Here $\phi(0)$ is the operator corresponding to the state $|\phi\rangle$ through the operator-state correspondence, and $f \circ \phi(0)$ denotes its conformal transformation. The operators

$$
U_{f}=e^{\sum_{n \geq 0} v_{n} L_{n}}
$$

satisfying

$$
f \circ \phi(z)=U_{f} \phi(z) U_{f}^{-1}, \quad \forall \phi
$$

form a representation of the conformal group on the Hilbert space of the worldsheet CFT. The coefficients $v_{n}$ in front of the Virasoro operators $L_{n}$ can be determined by solving certain recursive equations. The vector field $v(z)=\sum v_{n} z^{n+1}$ is also determined up to a constant by the equation $v(z) \partial f=v(f(z))$. For recent review and discussion of related issues the reader is referred to [14].

Using conformal map

$$
h(z)=\frac{1+i z}{1-i z}
$$

which maps the upper half plane with a coordinate $z$ one to one onto the unit disk with a coordinate $w=h(z)$ we can evaluate the correlator (30) as

$$
\langle f \circ \phi(0)\rangle_{\mathrm{UHP}}=\langle h \circ f \circ \phi(0)\rangle_{\text {disk }}
$$

The image of the unit upper half disk under the map $h \circ f$ is called local coordinate patch. For the simplest butterfly surface state corresponding to the map (29) with $r=2$ the shape of the local patch is indicated in Fig. 1. Recall, that in this coordinate system the local coordinate patch for the sliver state looks just as a sliver.

There is another coordinate system with a coordinate $\hat{w}=h \circ f^{-1} \circ h^{-1}(w)$, in which the local patch is mapped to the right half disk. In this coordinate system the star 
product of surface states is represented simply by gluing the surfaces with the local patch detached. For the butterfly state, the part of the surface without the local patch looks as a butterfly, hence the name. For the sliver the surface in this coordinates looks as a helix of an infinite excess angle.

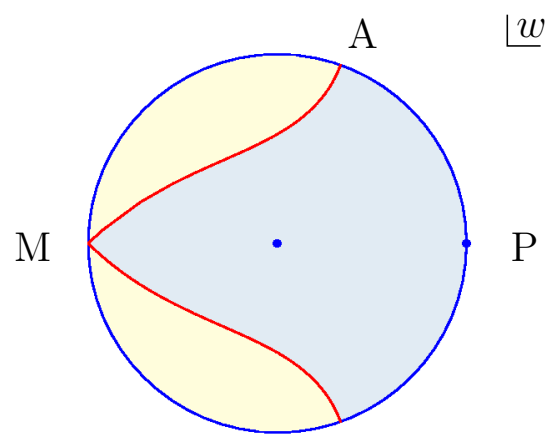

$\mathrm{B}$

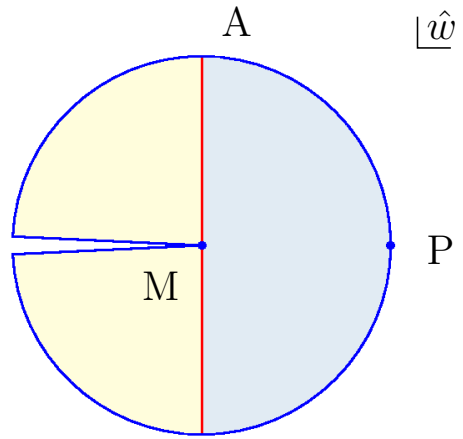

$\mathrm{B}$

Figure 1: Representation of the butterfly state $\langle 0| e^{-\frac{1}{2} L_{2}}$ in two coordinate systems. The local coordinate patch around the puncture $P$ is indicated by the darker shade. In the $\hat{w}$ coordinate system the local patch is represented always as the right half disk. The rest of the surface in this case is a half disk cut into two parts along the line $[-1,0]$.

For general $r$, the butterfly states can be expressed explicitly as elements of the Verma module (representation space of the Virasoro algebra)

$$
|B, r\rangle=U_{r}^{\dagger} e^{-\frac{1}{2} L_{-2}}|0\rangle
$$

where $U_{r}^{\dagger}$ are well known from the studies of wedge states $[18,14]$. They are given by

$$
U_{r}^{\dagger}=e^{2 \log \frac{r}{2} A^{\dagger}}
$$

where

$$
A=-\frac{1}{2} L_{0}+\sum_{k=1}^{\infty} \frac{(-1)^{k}}{(2 k-1)(2 k+1)} L_{2 k}
$$

and the dagger denotes the BPZ conjugation $L_{n}^{\dagger}=(-1)^{n} L_{-n}$. Similar vector field has been also considered recently in [21].

From the proof we are going to present in the next subsection it follows, that the family of butterfly projectors can be generalized also to include surface states

$$
\left|B^{(2 k)}\right\rangle=e^{\frac{(-1)^{k}}{2 k} L_{-2 k}}|0\rangle
$$



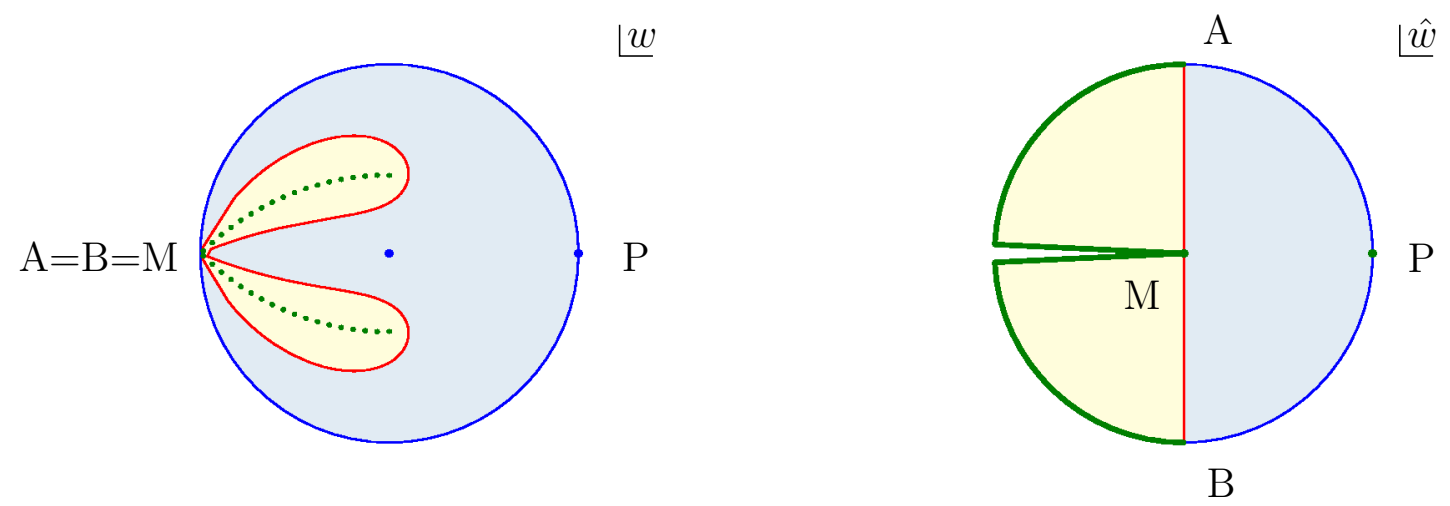

Figure 2: Representation of the butterfly state $\langle 0| e^{\frac{1}{4} L_{4}}$ in two coordinate systems. In the $\hat{w}$ coordinate system the local patch is represented by the right half disk, the rest of the surface on the right is cut along the line $[-1,0]$. Moreover the upper left $90^{\circ}$ arc is identified with the upper part of the cut and the lower left arc with the lower part of the cut.

associated to conformal maps

$$
f(z)=\frac{z}{\sqrt[2 k]{1-(-1)^{k} z^{2 k}}}
$$

considered already in [23]. An example of the corresponding surface for $k=2$ is given at Fig. 2.

These are direct generalizations of the state (35) for $r=2$. Once we establish that the states with $r=2$ are projectors, then the case $r \neq 2$ follows by considering finite reparametrizations and will be discussed separately in subsection 3.5.

\subsection{Proof that butterflies are projectors}

Let us assume for simplicity, that our CFT has overall central charge $c=0$. The complications for nonzero central charge, as in the matter plus twisted ghost CFT can be dealt with separately. We start then with the following simple identity

$$
e^{\alpha K_{2 k}}=e^{-\frac{1}{2 k} \tanh 2 k \alpha L_{-2 k}}(\cosh 2 k \alpha)^{-\frac{1}{k} L_{0}} e^{\frac{1}{2 k} \tanh 2 k \alpha L_{2 k}}
$$

discussed more in Appendix A. Using this formula we can write the butterfly state in a regulated form as

$$
e^{(-)^{k+1} \alpha K_{2 k}}|0\rangle=e^{\frac{(-1)^{k}}{2 k} \tanh 2 k \alpha L_{-2 k}}|0\rangle,
$$


where $\alpha \rightarrow \infty$. The advantage of this expression for the butterfly is that $K_{2 k}$ are (exterior) derivations of the star algebra and thus we have in general

$$
e^{K}(|\phi\rangle *|\chi\rangle)=e^{K}|\phi\rangle * e^{K}|\chi\rangle
$$

for any two string fields $\phi$ and $\chi$.

To prove that the butterfly is a projector we can write

$e^{\frac{(-1)^{k}}{2 k} L_{-2 k}}|0\rangle * e^{\frac{(-1)^{k}}{2 k} L_{-2 k}}|0\rangle=\lim _{\alpha \rightarrow \infty} e^{(-)^{k+1} \alpha K_{2 k}}|0\rangle * e^{(-)^{k+1} \alpha K_{2 k}}|0\rangle=\lim _{\alpha \rightarrow \infty} e^{(-)^{k+1} \alpha K_{2 k}} U_{3}^{\dagger}|0\rangle$.

Now formally holds

$$
\lim _{\alpha \rightarrow \infty} e^{(-)^{k+1} \alpha K_{2 k}} U_{3}^{\dagger}|0\rangle=\lim _{\alpha \rightarrow \infty} e^{\frac{(-1)^{k}}{2 k} L_{-2 k}}(\cosh 2 k \alpha)^{-\frac{1}{k} L_{0}} e^{-\frac{(-1)^{k}}{2 k} L_{2 k}} U_{3}^{\dagger}|0\rangle=e^{\frac{(-1)^{k}}{2 k} L_{-2 k}}|0\rangle,
$$

since all the terms in $e^{-\frac{(-1)^{k}}{2 k} L_{2 k}} U_{3}^{\dagger}|0\rangle$ with nonzero level get multiplied with vanishing factors. There is one important potential flaw in this argument however. We could have repeated all the argument without bothering about the $(-1)^{k}$ factor. ${ }^{3}$ This would imply the incorrect statement that $e^{\frac{1}{2} L_{-2}}|0\rangle$ is also a projector. What goes wrong in this case is that $e^{-\frac{1}{2} L_{2}} U_{3}^{\dagger}|0\rangle$ is divergent in the level expansion. When regulated, the divergences are just cancelled by the vanishing factors and we end up with something finite.

To complete the proof we have to show that

$$
\lim _{\alpha \rightarrow \infty} e^{-\frac{(-1)^{k}}{2 k} \tanh 2 k \alpha L_{2 k}} U_{3}^{\dagger}|0\rangle=e^{-\frac{(-1)^{k}}{2 k} L_{2 k}} U_{3}^{\dagger}|0\rangle
$$

has finite unambiguous limit. For $k=1$ it can be proved by explicit calculation (see below), for general $k$ we have to use the gluing theorem.

In general for two conformal maps $f$ and $g$ the state $\langle 0| U_{f} U_{g}^{\dagger}$ is again a surface state. It can be calculated as follows: From the surfaces corresponding to the states $\langle 0| U_{f}$ and $\langle 0| U_{g}$ in the $\hat{w}$ coordinate we detach the local coordinate patches and glue the remaining surfaces together along the segment $A B M$. The part of the surface corresponding to $\langle 0| U_{g}$ represents now new coordinate patch and we may map it back to the canonical half-disk. The resulting surface is then the surface representation of

\footnotetext{
${ }^{3}$ We learned about the subtleties related to this factor from Barton Zwiebach.
} 

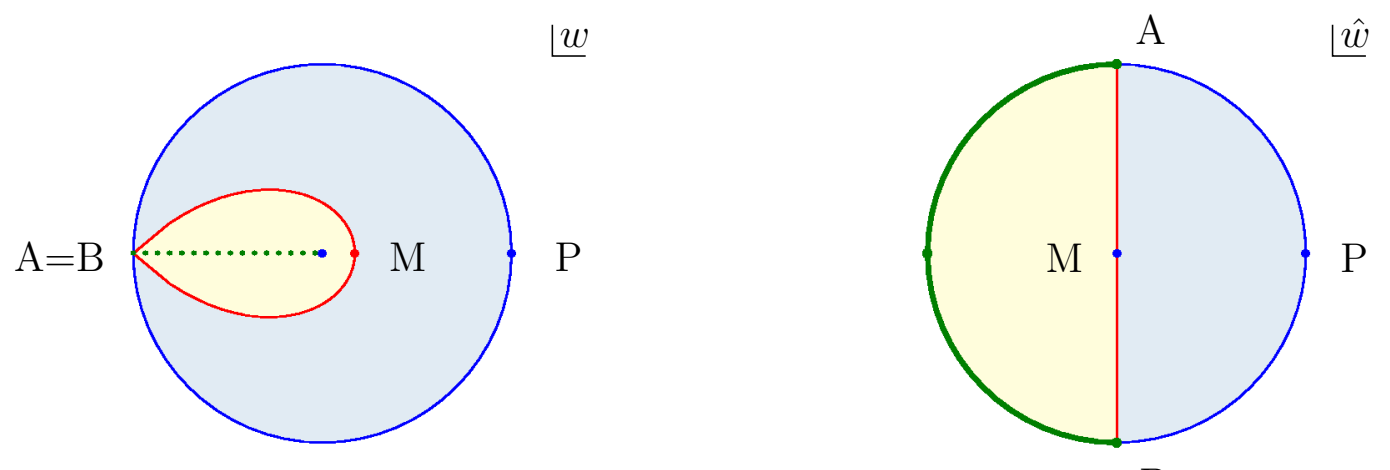

$\mathrm{B}$

Figure 3: Representation of the 'wrong sign' butterfly state $\langle 0| e^{\frac{1}{2} L_{2}}$. In the $\hat{w}$ coordinate system the upper and lower halves of the left semicircle, indicated by thick line, are identified. Note that the surface is completely regular at the midpoint.

the state $\langle 0| U_{f} U_{g}^{\dagger}$. This procedure has been applied to the case of wedge states in [14], where it was shown to agree with other methods.

In our present case we have to glue a wedge with opening angle $2 \pi$ (after detaching the local coordinate patch) with a surface associated to the map

$$
f(z)=\frac{z}{\sqrt[2 k]{1+(-1)^{k} \tanh (2 k \alpha) z^{2 k}}}
$$

Two examples of these surfaces are in Fig. 3 and 4 . Since this map is regular at the midpoint $M$, which in the upper half plane coordinates is located at $z=i$ the associated surface is regular at this point. This is also seen at the pictures Fig. 3 and 4 but is manifestly not true for the surfaces associated to butterfly states at Fig. 1 and 2. The midpoint $M$ is the only point where the wedge corresponding to $\langle 3|$ has a singularity. Gluing the wedge with the 'wrong sign' butterfly surface associated to the map (46) results in a surface with simple singularities which are just cuts and conical singularities which can be smoothed out. This proves that the state (45) is finite and well defined state and concludes thus the proof.

On the other hand, changing the sign in the exponent of the butterfly states would violate the last argument. We would have to glue two surfaces, one which has a cut originating at the midpoint $M$ and other which has a conical singularity there. This is a source of divergences which cancel the vanishing factors in (44). For instance the product like $e^{\frac{1}{2} L_{-2}}|0\rangle * e^{\frac{1}{2} L_{-2}}|0\rangle$ is finite and well defined but does not lead to $e^{\frac{1}{2} L_{-2}}|0\rangle$. 


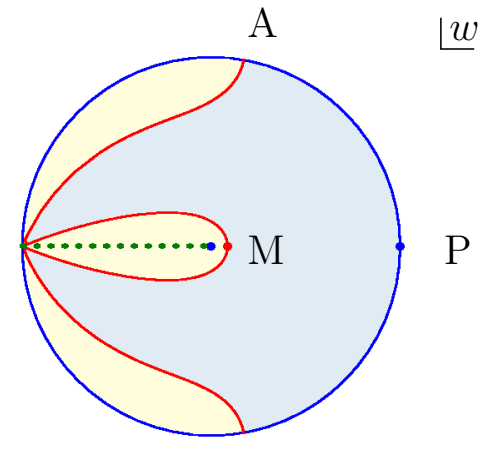

$\mathrm{B}$

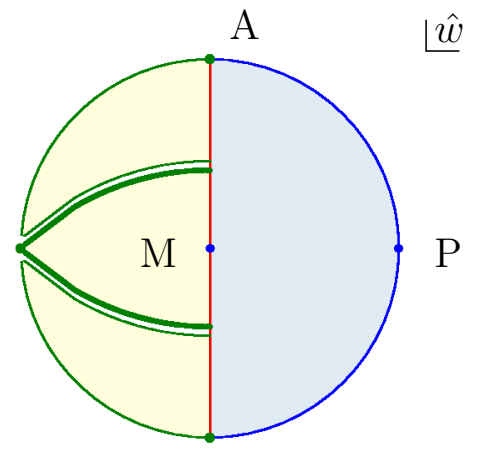

$\mathrm{B}$

Figure 4: Representation of the 'wrong sign' butterfly state $\langle 0| e^{-\frac{1}{4} L_{4}}$. In the $\hat{w}$ coordinate system the surface without the local patch is split into three independent parts, the middle part has its boundaries identified. Again the surface is perfectly regular at the midpoint.

\subsection{Some explicit calculations}

Using the conservation laws (137) and the methods of [14] we may find

$$
U_{r} e^{\frac{1}{2} \tan ^{2} \gamma L_{-2}}|0\rangle=\left(\frac{2}{r}\right)^{-\frac{c}{16}}\left(\frac{\cos \frac{2 \gamma}{r}}{\cos \gamma}\right)^{-\frac{3 c}{16}}\left(\frac{\sin \frac{2 \gamma}{r}}{\sin \gamma}\right)^{\frac{c}{16}} e^{\frac{1}{2} \tan ^{2} \frac{2 \gamma}{r} L_{-2}}|0\rangle .
$$

We have written the formula for general central charge $c$, now we shall restrict to the case $c=0$ however. In general, if we know explicitly the surface state $U_{f} U_{g}^{\dagger}|0\rangle=$ $U_{\phi[f, g]}^{\dagger}|0\rangle$ we may calculate $U_{g} U_{f}^{\dagger}|0\rangle=U_{\phi[g, f]}^{\dagger}|0\rangle$ using

$$
\phi[g, f]=I \circ \phi[f, g] \circ I \circ f \circ I \circ g^{-1} \circ I .
$$

This can be easily established by calculating $\left\langle 0\left|\Psi(z) U_{f} U_{g}^{\dagger}\right| 0\right\rangle$ for an arbitrary local field $\Psi(z)$. There are two different ways of bringing it to the form $\left\langle f_{1,2} \circ \Psi(z)\right\rangle$ whose equivalence requires (48).

Note that with our definition the functions $\phi[f, g]$ and $\phi[g, f]$ are defined modulo $S L(2, \mathbb{C})$ transformation acting from the left, which is immaterial for the definition of the surface states. There is one preferred choice however and this is the reason why the first factor on the right hand side of (48) is $I$. With this choice we have

$$
U_{f} U_{g}^{\dagger}=U_{\phi[f, g]}^{\dagger} U_{\phi[g, f]}
$$


as the operator statement and for the corresponding maps

$$
f \circ I \circ g^{-1} \circ I=I \circ \phi[f, g]^{-1} \circ I \circ \phi[g, f] .
$$

The equation (49) has been used in [25] for the algebraic proof of the gluing theorem. The maps $\phi[f, g]$ and $\phi[g, f]$ were constructed as series in an auxiliary parameter $\alpha$ which controls the contribution of higher levels and which has to be sent to 1 at the end of the calculation. Taking

$$
f(z)=\tan \left(\frac{2}{r} \arctan z\right), \quad g(z)=\frac{z}{\sqrt{1-\tan ^{2} \gamma z^{2}}}
$$

and using (47), (48) and (136) we find easily

$$
\begin{aligned}
\phi[f, g](z) & =\frac{z}{\sqrt{1-\tan ^{2}\left(\frac{2 \gamma}{r}\right) z^{2}}} \\
\phi[g, f](z) & =\sqrt{\tan ^{2}\left(\frac{2}{r} \arctan \sqrt{z^{2}+\tan ^{2} \gamma}\right)-\tan ^{2} \frac{2 \gamma}{r}} .
\end{aligned}
$$

Specializing to $\gamma=\frac{\pi}{4}$ and $r=3$ we find by expanding (53) in series and solving iteratively for the generating vector field as in [18]

$$
e^{\frac{1}{2} L_{2}} U_{3}^{\dagger}|0\rangle=e^{\frac{-3+\sqrt{3}}{12} L_{-2}+\frac{1}{144} L_{-4}+\frac{-27+7 \sqrt{3}}{1552} L_{-6}+\frac{3-\sqrt{3}}{7776} L_{-8}+\cdots}|0\rangle .
$$

This supports our claim that it is a well defined state with finite coefficients and therefore the butterfly state $e^{-\frac{1}{2} L_{-2}}|0\rangle$ is a true projector.

Let us now look at what happens to the wrong sign butterfly states under the star product. The function (53) clearly ceases to be holomorphic at $z=0$ in the limit $\gamma \rightarrow i \infty$ for $r=3$ and the coefficients of the corresponding surface state diverge. Multiplying the state by the middle factor in (40) which is now

$$
\left(1-(\tan \gamma)^{4}\right)^{\frac{L_{0}}{2}} \approx\left(\frac{\sqrt{2}}{\cosh |\gamma|}\right)^{L_{0}}
$$

has the effect of changing $z \rightarrow \frac{\sqrt{2} z}{\cosh |\gamma|}$ in (53), which in our limit keeps the domain of holomorphicity around zero from shrinking to zero size. Up to an irrelevant overall factor the function (53) with this substitution has a well defined limit

$$
\sqrt{\left(1+2 z^{2}\right)^{\frac{2}{3}}-1}
$$


Finally multiplying the state with $e^{\frac{1}{2} L_{-2}}$ gives a surface state associated to the map

$$
\sqrt{\left(\frac{1+z^{2}}{1-z^{2}}\right)^{\frac{2}{3}}-1}
$$

Using the recursive relations we determine again the generating vector field and the coefficients in front of the Virasoro generators. The result is

$$
e^{\frac{1}{2} L_{-2}}|0\rangle * e^{\frac{1}{2} L_{-2}}|0\rangle=e^{\frac{1}{3} L_{-2}+\frac{5}{54} L_{-4}-\frac{5}{162} L_{-6}+\frac{59}{2916} L_{-8}+\cdots}|0\rangle .
$$

Results from independent numerical check at level 16 are summarized in Appendix D. Note also that this state is annihilated by $K_{2}$ just as the false butterfly $e^{\frac{1}{2} L_{-2}}|0\rangle$ itself. If we keep multiplying these states we get a whole commutative subalgebra annihilated by $K_{2}$, which resembles in many respects the subalgebra of wedge states, which are characterized by the fact, that they are annihilated by $K_{1}$.

The calculation we have just performed can be easily generalized. From the formulas (40) and (53) we can calculate the star product of regularized butterflies. The star product

$$
e^{-\frac{1}{2} \tan ^{2} \gamma L_{-2}}|0\rangle * e^{-\frac{1}{2} \tan ^{2} \gamma L_{-2}}|0\rangle=U_{f_{3, \gamma}}^{\dagger}|0\rangle
$$

is a surface state associated to the map

$$
f_{3, \gamma}(z)=\sqrt{\tan ^{2}\left(\frac{2}{3} \arctan \sqrt{\left.\frac{z^{2}+\tan ^{2} \gamma}{1+z^{2} \tan ^{2} \gamma}\right)-\tan ^{2} \frac{2 \gamma}{3}}\right.}
$$

and therefore it looks as

$$
U_{f_{3, \gamma}}^{\dagger}|0\rangle=e^{v_{2} L_{-2}+v_{4} L_{-4}+\cdots}|0\rangle,
$$

where the first two coefficients are

$$
\begin{aligned}
& v_{2}=\frac{-3-4 \cos \frac{4 \gamma}{3}+2 \cos \frac{8 \gamma}{3}}{3\left(1+2 \cos \frac{4 \gamma}{3}\right)^{2}} \\
& v_{4}=\frac{\left(1-2 \cos \frac{4 \gamma}{3}\right)^{2}\left(47+60 \cos \frac{4 \gamma}{3}+10 \cos \frac{8 \gamma}{3}\right)}{54\left(1+2 \cos \frac{4 \gamma}{3}\right)^{4}} .
\end{aligned}
$$

The multiple star products

$$
\underbrace{e^{-\frac{1}{2} \tan ^{2} \gamma L_{-2}}|0\rangle * e^{-\frac{1}{2} \tan ^{2} \gamma L_{-2}}|0\rangle * \cdots * e^{-\frac{1}{2} \tan ^{2} \gamma L_{-2}}|0\rangle}_{(r-1) \text { times }}=U_{f_{r, \gamma}}^{\dagger}|0\rangle
$$


correspond to maps which look just as (60) with the two factors of 3 replaced by $r$. From the formula then follows that the limit of the repeated multiplication of $e^{\frac{1}{2} L_{-2}}|0\rangle$ with itself is just the true buttefly state $e^{-\frac{1}{2} L_{-2}}|0\rangle$.

\subsection{Near butterfly limit}

Let us consider now in detail the limit $\gamma \sim \frac{\pi}{4}$, in which the state $e^{-\frac{1}{2} \tan ^{2} \gamma L_{-2}}|0\rangle$ approaches the butterfly projector. The product of two such states is a surface state associated to the map

$$
f(z)=\left(\frac{4}{3}\right)^{\frac{5}{4}} \sqrt{\frac{\pi}{4}-\gamma}\left[\frac{z}{\sqrt{1+z^{2}}}+\frac{2}{\sqrt{3}}\left(\gamma-\frac{\pi}{4}\right) \frac{z}{\left(1+z^{2}\right)^{\frac{3}{2}}}+O\left(\gamma-\frac{\pi}{4}\right)^{2}\right] .
$$

Due to the fact that the second term in the bracket is just a change of the first under infinitesimal scaling proportional to

$$
z \frac{d}{d z}\left(\frac{z}{\sqrt{1+z^{2}}}\right)=\frac{z}{\left(1+z^{2}\right)^{\frac{3}{2}}}
$$

we find easily

$$
\begin{aligned}
e^{s L_{-2}}|0\rangle * e^{s L_{-2}}|0\rangle & =\left[1-\left(1-\frac{1}{\sqrt{3}}\right)\left(s+\frac{1}{2}\right) L_{-2}\right] e^{s L_{-2}}|0\rangle+O\left(s+\frac{1}{2}\right)^{2} \\
& =\left[1+\left(1-\frac{1}{\sqrt{3}}\right)\left(s+\frac{1}{2}\right) L_{0}\right] e^{s L_{-2}}|0\rangle+O\left(s+\frac{1}{2}\right)^{2}
\end{aligned}
$$

We thus see that $|\psi\rangle=e^{s L_{-2}}|0\rangle$ solves the equation of motion of ghost number zero string field theory

$$
|\psi\rangle *|\psi\rangle=\left(1+a^{-1} L_{0}\right)|\psi\rangle
$$

in the limit $a \rightarrow \infty$, provided we identify

$$
a^{-1}=\left(1-\frac{1}{\sqrt{3}}\right)\left(s+\frac{1}{2}\right) .
$$

This can be directly extended to the case of standard regularized VSFT, where up to terms of order $O\left(s+\frac{1}{2}\right)^{2}$ the solution is

$$
\left|\Psi_{0}\right\rangle=\mathcal{N} e^{s\left(L_{-2}^{X}+L_{-2}^{\prime g h}\right)} c_{1}|0\rangle
$$

with the relation between $a$ and $s$ being the same as in (68). The normalization factor $\mathcal{N}$ is for $s \neq-\frac{1}{2}$ finite and calculable. We have thus fully proven the conjecture of [7] for which we gave a lot of evidence in section 2.3. 


\subsection{Example of divergent star products}

Now we would like to prove our claim from section 2.2 that the star product $|B\rangle * L_{-2}^{X}|B\rangle$ is ill defined. To demonstrate it, we shall calculate the even level part of the regulated expression

$$
L_{-2}^{X} e^{\alpha K_{2}}|0\rangle * e^{\alpha K_{2}}|0\rangle
$$

and show that it is divergent in the limit $\alpha \rightarrow \infty$. From (137) follows

$$
L_{-2}^{X} e^{\alpha K_{2}}|0\rangle=-\cosh ^{2} 2 \alpha\left(K_{2}^{X}+\frac{c}{4} \tanh 2 \alpha\right) e^{\alpha K_{2}}|0\rangle .
$$

Using the $K_{2}^{X}$ conservation law and (42) we get

$$
\begin{aligned}
& L_{-2}^{X} e^{\alpha K_{2}}|0\rangle * e^{\alpha K_{-2}}|0\rangle+e^{\alpha K_{-2}}|0\rangle * L_{-2}^{X} e^{\alpha K_{-2}}|0\rangle= \\
& \quad=-\cosh ^{2} 2 \alpha\left[K_{2}^{X}+\frac{c}{2} \tanh 2 \alpha-3 k_{2}\right] e^{\alpha K_{2}} U_{3}^{\dagger}|0\rangle .
\end{aligned}
$$

Using the relation (44) we arrive to

$$
\begin{gathered}
\lim _{\alpha \rightarrow \infty} \frac{1}{\cosh ^{2} 2 \alpha}\left[L_{-2}^{X} e^{-\frac{1}{2} \tanh 2 \alpha L_{-2}}|0\rangle * e^{-\frac{1}{2} \tanh 2 \alpha L_{-2}}|0\rangle+\right. \\
\left.e^{-\frac{1}{2} \tanh 2 \alpha L_{-2}}|0\rangle * L_{-2}^{X} e^{-\frac{1}{2} \tanh 2 \alpha L_{-2}}|0\rangle\right]=c\left(-\frac{1}{4}-\frac{65}{9}\right) e^{-\frac{1}{2} L_{-2}}|0\rangle
\end{gathered}
$$

demonstrating that the star product in the square bracket is indeed divergent in the limit $\alpha \rightarrow \infty$.

\subsection{Family of butterfly states}

To show that also the whole family of butterfly states

$$
|B, r\rangle=U_{r}^{\dagger} e^{-\frac{1}{2} L_{-2}}|0\rangle
$$

are projectors is rather easy. Important observation is that $A$ given by (37) annihilates the simplest butterfly

$$
A e^{-\frac{1}{2} L_{-2}}|0\rangle=0
$$

thanks to the identity

$$
\sum_{k=1}^{\infty} \frac{1}{(2 k-1)(2 k+1)}=\frac{1}{2}
$$


Therefore, using the formula

$$
e^{-\alpha\left(A-A^{\dagger}\right)}=U_{1+e^{\alpha}}^{\dagger} U_{1+e^{-\alpha}}
$$

from [14] we get

$$
|B, r\rangle=e^{-\log (r-1)\left(A-A^{\dagger}\right)} e^{-\frac{1}{2} L_{-2}}|0\rangle .
$$

The exponent $D \equiv A-A^{\dagger}$ is an exterior derivative in the algebra (it is a linear combination of $K_{n}$ 's) and therefore one may use (42) to show that all members of the butterfly family are projectors and all arise as reparametrizations of the vacuum. Special cases are the $r \rightarrow \infty$ limit, which gives the sliver, and the $r \rightarrow 1$ limit leading to an interesting state which resembles the identity of [24]

$$
|B, 1\rangle=\left(\prod_{n=2}^{\infty} e^{-\frac{2}{2^{n}} L_{-2^{n}}}\right) e^{-L_{-2}}|0\rangle .
$$

It differs from the identity only in the sign of $L_{-2}$ in the exponent.

\section{On the uniqueness of the projector}

In section 2 we have shown that the simplest butterfly projector

$$
\left|\Psi_{0}\right\rangle=\mathcal{N} e^{-\frac{1}{2}\left(L_{-2}^{X}+L_{-2}^{\prime g h}\right)} c_{1}|0\rangle
$$

does obey all the quadratic identities, which should be satisfied by all classical solutions of the regularized string field theory in a Siegel gauge. An immediate question that arises is, to what extent is this solution unique. We are going to prove, that in the space of surface states the identities are so constraining that the solution (80) is indeed a unique solution.

It turns out, that we do not need to study the whole set of identities, we will see that already in the matter sector alone the constraint

$$
\left\langle\Psi_{0}\left|L_{2 k}\right| \Psi_{0}\right\rangle \sim(-1)^{k}
$$

is strong enough to disqualify all other surface states as possible solutions. The symbol $\sim$ means up to a possibly divergent normalization constant independent of $k$. 
Before we start the actual proof, we can easily demonstrate that the generalized butterfly projectors certainly do not obey the identities. Indeed, for $n>2$ even

$$
\left\langle 0\left|e^{s L_{n}} L_{m} e^{s L_{-n}}\right| 0\right\rangle=0 \quad \forall m \in \mathbb{Z} \backslash\{\ldots,-n, 0, n, 2 n, 3 n, \ldots\}
$$

but

$$
\left\langle 0\left|e^{s L_{n}} L_{n} e^{s L_{-n}}\right| 0\right\rangle=\frac{c}{12}\left(n^{3}-n\right) s\left(1-(n s)^{2}\right)^{-\frac{c}{12} \frac{n^{2}-1}{n}-1} \neq 0 .
$$

The reason is that if $m$ is not an integer multiple of $n$, after normal ordering of the operators all the terms will have their $L_{0}$ weight nonzero, equal to $m$ modulo $n$ and therefore the expectation value will vanish.

\subsection{Testing the identities for surface states}

Let us now describe a general method of testing the identity (81) applicable to any surface state

$$
\left|\Psi_{0}\right\rangle=U_{f}^{\dagger}|0\rangle
$$

associated to a given conformal map $f(z)$. We are going to calculate the matrix elements

$$
\left\langle 0\left|U_{f} L_{-m} U_{f}^{\dagger}\right| 0\right\rangle=\oint \frac{d z}{2 \pi i} \frac{1}{z^{m-1}}\left\langle 0\left|U_{f} T(z) U_{f}^{\dagger}\right| 0\right\rangle .
$$

From the geometric discussion in section 3 the state $\langle 0| U_{f}$, which formally exists in the Verma module for any $f(z)$ holomorphic near origin, is meaningful in the CFT sense only if $f(z)$ maps one to one the upper unit half disk into the upper half plane. The projectors which seem to be characterized by the fact that $f(i) \rightarrow \infty$ are thus limiting cases. The identity and the false butterflies with $f( \pm 1) \rightarrow \infty$ are also limits of regular surface states. We shall therefore restrict to the regular surface states only and later discuss these interesting limits.

We also have to require that

$$
\langle 0| U_{f} T(z)=\langle 0| f \circ T(z) U_{f}
$$

is a well defined state in the level expansion. This is not automatic, since the left hand side is a series in positive and negative powers of $z$. Negatively moded Virasoro 
generators when commuted to the left give rise to infinite series' in mostly positive powers of $z$, which then should sum to coefficients

$$
\frac{\left(f^{\prime}(z)\right)^{2}}{f(z)^{m+2}} \quad m \in \mathbb{N} .
$$

The convergence of these Verma module coefficients follows from our assumptions about the map $f(z)$ provided $|z|<R$, where $R>1$ is the radius inside which the map is holomorphic and one to one into the upper half plane. Since we need also $T(z) U_{f}^{\dagger}|0\rangle$ being well defined, this implies $|z|>1 / R$. In order for $\left\langle 0\left|U_{f} T(z) U_{f}^{\dagger}\right| 0\right\rangle$ to be absolutely convergent we need thus

$$
|z| \in\left(R^{-1}, R\right) \quad \text { and } \quad R>1 \text {. }
$$

From what has been said now follows that the right location of the contour in (85) is inside the annulus of radii $1 / R$ and $R$. To calculate the integrand we need to use the transformation properties of the energy momentum tensor $T(z)$

$$
f \circ T(z)=\left(f^{\prime}(z)\right)^{2} T(f(z))+\frac{c}{12} S[f, z]
$$

where

$$
S[f, z]=\frac{\partial^{3} f \partial f-\frac{3}{2}\left(\partial^{2} f\right)^{2}}{(\partial f)^{2}}
$$

is the Schwarzian derivative. We can then calculate

$$
\begin{aligned}
& \left\langle 0\left|U_{f} T(z) U_{f}^{\dagger}\right| 0\right\rangle=\left\langle 0\left|f \circ T(z) U_{f} U_{f}^{\dagger}\right| 0\right\rangle=e^{c \kappa}\left\langle 0\left|f \circ T(z) U_{\phi[f, f]}^{\dagger}\right| 0\right\rangle= \\
& =e^{c \kappa}\langle 0|I \circ \phi \circ I \circ f \circ T(z)| 0\rangle=\frac{c}{12} S[I \circ \phi \circ I \circ f, z] e^{c \kappa},
\end{aligned}
$$

where the function $\phi \equiv \phi[f, f]$ was introduced and discussed in section 3.2. The central charge $c$ appearing in the normalization constant $e^{c \kappa}=\left\langle 0\left|U_{f} U_{f}^{\dagger}\right| 0\right\rangle$ might in principle be different from that of the energy momentum tensor we insert, but that is just a trivial extension of our formulas.

Before we move on to the proof, let us have a look on two examples of the butterfly and sliver projectors. 


\subsubsection{Butterfly example}

For regularized butterfly state associated to the function

$$
f_{s}(z)=\frac{z}{\sqrt{1-2 s z^{2}}}
$$

the function $\phi=\phi[f, f]$ is given by

$$
\phi(z)=\frac{z}{\sqrt{\left(1-4 s^{2}\right)-2 s z^{2}}} .
$$

and

$$
I \circ \phi \circ I \circ f(z)=\sqrt{\frac{z^{2}-2 s}{1-2 s z^{2}}} .
$$

The Schwarzian derivative is then

$$
S[I \circ \phi \circ I \circ f, z]=-\frac{3}{2 z^{2}}\left(1+\frac{1-4 s^{2}}{\left(z^{2}-2 s\right)^{2}}\right)\left(1-\frac{1-4 s^{2}}{\left(1-2 s z^{2}\right)^{2}}\right) .
$$

We thus see that the contour integral in (85) receives contribution from poles at $0, \pm \sqrt{2 s}$. Up to a normalization constant $\left\langle 0\left|U_{f} U_{f}^{\dagger}\right| 0\right\rangle$ it is equal to

$$
\frac{c}{12} \oint \frac{d z}{2 \pi i} \frac{1}{z^{2 k-1}} S[I \circ \phi \circ I \circ f, z]=c(2 s)^{k}\left(\frac{s^{2}}{1-4 s^{2}}+\frac{k+1}{8}\right),
$$

for $m=2 k$ even. For $m$ odd the integral vanishes. Although the above calculation is rather straightforward, these results can be obtained in a much simpler way by working directly with the Virasoro generators and conservation laws as we discuss in the appendices B and C. Notice also that in the limit $s \rightarrow-\frac{1}{2}$ the integral behaves like $(-1)^{k}$ and we readily confirm the identities (81).

\subsubsection{Sliver example}

The situation for the sliver is more complicated. Even if we regularize the sliver by considering wedge state associated to the map

$$
f_{r}(z)=\tan \left(\frac{2}{r} \arctan z\right)
$$

for large but finite $r$, this function still has branch cuts starting at $\pm i$ and going all the way to infinity. Therefore the expression $\left\langle 0\left|U_{f} T(z) U_{f}^{\dagger}\right| 0\right\rangle$ does not absolutely converge 
for any values of $z$. In the limit $r \rightarrow \infty$ we can however circumvent this problem quite unambiguously as we shall see.

The Schwarzian derivative for the wedge states reads

$$
S\left[f_{r}, z\right]=\frac{4-r^{2}}{r^{2}} \frac{2}{\left(1+z^{2}\right)^{2}}
$$

and the function $\phi_{r}=\phi\left[f_{r}, f_{r}\right]$ is given by

$$
\phi_{r}(z)=f_{4-\frac{4}{r}}(z)
$$

as was found in [14]. Let us start by calculating

$$
\langle 0| U_{f} L_{-m}=\frac{c}{12} \oint \frac{d z}{2 \pi i} \frac{1}{z^{m-1}} S[f, z]\langle 0| U_{f}+\oint \frac{d z}{2 \pi i} \frac{1}{z^{m-1}}\left(f^{\prime}(z)\right)^{2}\langle 0| T(f(z)) U_{f} .
$$

The contour encircles the origin and remains inside the unit circle. Then we contract the expression with $U_{f}^{\dagger}|0\rangle$. Up to the overall factor $\left\langle 0\left|U_{f} U_{f}^{\dagger}\right| 0\right\rangle$ the first term gives

$$
\frac{c}{12} \oint \frac{d z}{2 \pi i} \frac{1}{z^{m-1}} S[f, z]
$$

which is fine as far as concerns the integral. The second term is then

$$
\frac{c}{12} \oint \frac{d z}{2 \pi i} \frac{1}{z^{m-1}} \frac{\left(f^{\prime}(z)\right)^{2}}{f(z)^{4}} S[\phi, I \circ f(z)] .
$$

Note that the form of both terms can be understood as a consequence of the identity for the Schwarzian derivative

$$
S[I \circ \phi \circ I \circ f, z]=S[f, z]+\frac{\left(f^{\prime}(z)\right)^{2}}{f(z)^{4}} S[\phi, I \circ f(z)],
$$

but we have chosen the stepwise derivation. The location of the contour in the second term is ambiguous. In fact it cannot be deformed in any way to ensure the absolute convergence. We may take it along the unit circle, but then have to assume some additional convenient regularization for all $r$. What saves us in the case of sliver is that

$$
\frac{\left(f^{\prime}(z)\right)^{2}}{f(z)^{4}} S[\phi, I \circ f(z)]=-2 \frac{(r-2)(3 r-2)}{r^{2}(r-1)^{2}}\left(\frac{1}{1+z^{2}}\right)^{2}
$$


uniformly vanishes for $r \rightarrow \infty$. Whatever $r$ independent regularization we decide for, the second term in the limit will not contribute. Therefore a unique contribution comes from the first term (100) and we find for the sliver $|\Xi\rangle=\lim _{r \rightarrow \infty} U_{r}^{\dagger}|0\rangle$

$$
\left\langle\Xi\left|L_{2 k}\right| \Xi\right\rangle=\frac{c}{6} \cdot k(-1)^{k}\langle\Xi \mid \Xi\rangle
$$

which is clearly different from (81). The normalization factor $\langle\Xi \mid \Xi\rangle$, which is divergent has been also calculated in [14].

\subsection{Proof of uniqueness among surface states}

We shall start by rewriting the set of quadratic identities (9) or (21) in a compact form. Consider a variation

$$
\delta \Psi=\left(T(z)-\frac{1}{z^{4}} T(-1 / z)\right) \Psi_{0}
$$

around a solution $\Psi_{0}$ of the equations of motion of a given string field theory. Here $T(z)$ stands for any of the matter, ghost, twisted ghost energy momentum tensors or combinations thereof. For any of these choices we have

$$
\left[L_{0}^{t o t}, T(z)\right]=\left(2+z \frac{d}{d z}\right) T(z) .
$$

The cubic term in the string field theory action is left invariant under the variation (105). This can be understood in two ways: First, this variation is an infinite sum of variations (8), in which the anomalies happen to cancel for a natural choice of regularization. Second, the invariance of the cubic term can be derived from the overlap equations for the three vertex [26]

$$
\left[T^{(s)}(z)-\frac{1}{z^{4}} T^{(s+1)}(-1 / z)\right]\left|V_{3}\right\rangle=0 .
$$

They are strictly speaking true only for $|z|=1, z \neq \pm i$ and with the prescription that in the first term $z$ approaches the unit circle from outside, whereas in the second term from interior. When contracted with regular states, these stringent conditions can be relaxed to some annulus around the unit circle.

As a consequence of the equations of motion, if the cubic term is invariant under the variation, so has to be also the kinetic term. From that we find an important relation

$$
\left(2+z \frac{d}{d z}\right)\left\langle\Psi_{0}|T(z)| \Psi_{0}\right\rangle=0
$$


whose unique solution is

$$
\left\langle\Psi_{0}|T(z)| \Psi_{0}\right\rangle=\frac{\lambda}{z^{2}}
$$

This equation contains as much information as the original set of identities (9) or (21). The constant $\lambda$ depends on the type of the energy momentum tensor and is also related to the anomaly. Details depend also on whether we are in vacuum or ordinary string field theory.

Now we are going to solve the equation (109) in the space of surface states discussed in section 4.1. Due to (90) our problem reduces to the equation

$$
S[I \circ \phi \circ I \circ f, z]=\frac{\mu}{z^{2}},
$$

where we denote

$$
\mu=\frac{12 \lambda}{c} e^{-c \kappa}
$$

Although the equation (110) looks as a third order nonlinear differential equation we can find easily all its solutions. Denoting

$$
g(z)=I \circ \phi \circ I \circ f(z)
$$

we find the solution

$$
\begin{aligned}
& g(z)=h\left(z^{\sqrt{1-2 \mu}}\right) \quad \text { for } \quad \mu \neq \frac{1}{2}, \\
& g(z)=h(\log z) \quad \text { for } \quad \mu=\frac{1}{2},
\end{aligned}
$$

where $h(z)$ is an arbitrary $S L(2, \mathbb{R})$ map. Note that for this reason the sign of the square root does not matter. The map $h(z)$ is parameterized by three real parameters, just as many as the number of parameters necessary to specify the initial conditions of a third order differential equation.

So we have managed to reduce the problem to the equation

$$
I \circ \phi \circ I \circ f(z)=h \circ z^{n}
$$

restricting for simplicity to the case $\mu \neq \frac{1}{2}$ and denoting $n=\sqrt{1-2 \mu}$. Now, a bit surprisingly the equation (115), for $n$ even at least, does not admit any regular solution. To see that, note that from (48) the left hand side of (115) commutes with the inversion

$$
I \circ(I \circ \phi \circ I \circ f)=(I \circ \phi \circ I \circ f) \circ I,
$$


but the right hand side never commutes, due to the fact that

$$
I \circ h=h \circ I \circ(-\mathrm{id})
$$

can be never satisfied within $S L(2, \mathbb{R})$ group. What we can do however, is to find a sequence of approximations $g_{n, a}(z)$ for $a \rightarrow 1$ whose limit is $g(z)=h \circ z^{n}$ and hope that the corresponding sequence of $f_{n, a}$ 's whose uniqueness we shall prove, have always the same limit. This is just a technical assumption of smooth dependence of $f$ on $g$.

The most natural sequence of approximations labelled with a continuous parameter $a$ is

$$
g_{n, a}(z)=\left(\frac{z^{n}+(-1)^{n} a}{1+a z^{n}}\right)^{\frac{1}{n}}
$$

For $n$ even and $a=1+\varepsilon$ we have

$$
g_{n, a}(z)=1+\frac{\varepsilon}{n} \cdot \frac{1-z^{n}}{1+z^{n}}+O(\varepsilon)^{2}
$$

and this has indeed the form of $h \circ z^{n}$ up to the first order in $\varepsilon$. We would have obtained a similar result also for $a$ close to -1 . The Schwarzian derivative (for all $n$ ) is

$$
S\left[g_{n, a}, z\right]=-\frac{n^{2}-1}{2 z^{2}}\left(1+\frac{1-a^{2}}{\left(z^{n}+(-1)^{n} a\right)^{2}}\right)\left(1-\frac{1-a^{2}}{\left(1+a z^{n}\right)^{2}}\right)
$$

and in the limit $a \rightarrow \pm 1$ it clearly approaches $-\frac{n^{2}-1}{2 z^{2}}$.

Now we are going to determine the surface states, which actually solve our equation (115) with $h \circ z^{n}$ replaced by $g_{n, a}(z)$. There are two ways to proceed. Based on our results from subsection 4.1.1, we could guess and check that (118) requires

$$
f(z)=\frac{z}{\left(1+a z^{n}\right)^{\frac{1}{n}}}
$$

and the limits $a \rightarrow \pm 1$ correspond to the butterfly and false butterfly states.

What about the uniqueness? It seems that we are getting more solutions depending on $n$, recall however, that different $n$ correspond to different $\lambda$ which we take fixed in such a way, that the butterfly state $e^{-\frac{1}{2} L_{-2}}|0\rangle$ solves the constraint. Are there any other solutions? We can prove uniqueness of the solution of the equation

$$
I \circ \phi \circ I \circ f(z)=g(z)
$$


for any given $g(z)$ by the following reasoning: Suppose there is another solution $\tilde{f}$ with $\tilde{\phi}=\phi[\tilde{f}, \tilde{f}]$ such that

$$
I \circ \phi \circ I \circ f=I \circ \tilde{\phi} \circ I \circ \tilde{f} .
$$

Since the operator corresponding to $I \circ \phi \circ I$ contains only negatively moded Virasoro operators, we find $\langle 0| U_{f}=\langle 0| U_{\tilde{f}}$ and thus $f(z)=\tilde{f}(z)$ modulo some unimportant global rescaling.

An alternative procedure is to recall from [22] that the map (118) is generated by a vector field $v(z)=z^{n+1}-(-)^{n} z^{-n+1}$, which obeys $v(I z)=\frac{1}{z^{2}} v(z) .{ }^{4}$ The operator which corresponds to the map $g_{n}$ is thus

$$
U_{g_{n}}=e^{\alpha K_{n}}
$$

The dependence of $\alpha$ on $s$ should be worked out separately. Again, since the operator corresponding to $I \circ \phi \circ I$ acts as identity on the vacuum $\langle 0|$ we have

$$
\langle 0| U_{f}=e^{\frac{c \kappa}{2}}\langle 0| e^{\alpha K_{n}},
$$

where $e^{c \kappa}=\left\langle 0\left|U_{f} U_{f}^{\dagger}\right| 0\right\rangle$. From the formulas in Appendix A we easily find the relation between the parameters $\alpha$ and $s$

$$
s=\frac{1}{2 k} \tanh 2 k \alpha .
$$

As a byproduct of the above arguments we obtain an interesting statement. For any regular surface states $\langle 0| U_{f}$ associated to the maps specified above, there exists a star algebra derivative $K$, which is a linear combination of the generators $K_{n}=$ $L_{n}-(-1)^{n} L_{-n}$, such that

$$
e^{K}=e^{-\frac{c \kappa}{2}} U_{\phi^{-1}}^{\dagger} U_{f}=e^{\frac{c \kappa}{2}} U_{\phi} U_{f^{-1}}^{\dagger}
$$

and therefore

$$
\langle 0| U_{f}=e^{\frac{c \kappa}{2}}\langle 0| e^{K} .
$$

This follows from the fact, that the function $g=I \circ \phi \circ I \circ f$ is generated by some $K$ and from the fact that $I \circ \phi \circ I \circ$ is holomorphic around $\infty$ and hence the corresponding operator acts trivially on $\langle 0|$. The equation (127) has been checked on a number of examples like wedge states in [14] and regularized butterflies in the present paper.

\footnotetext{
${ }^{4}$ In general any map $g$ satisfying $g \circ I=I \circ g$ is generated by a vector field $v(z)$ obeying $v(I z)=$ $\frac{1}{z^{2}} v(z)$, as can be seen from the equation $v(z) \partial g(z)=v(g(z))$.
} 


\section{Conclusions}

We have seen that the anomalous $K_{n}$ symmetries, which are just consequences of the overlap equations for the string vertex, yield nontrivial information about the solutions of the ordinary or vacuum string field theories.

In order to obtain finite action solutions in VSFT with pure ghost kinetic operator, we generically need infinite normalization of the action. Due to the singular nature of projectors it could happen that the normalization turns out to be finite. In any case it shows that some sort of regularization of the string field action is inevitable.

Solutions of the regularized version of VSFT constructed by adding a term $a^{-1} c_{0} L_{0}$ to the kinetic operator obey such a constraint, which among all the surface states admits unique solution which is just the simplest butterfly projector. The constraint, up to a single constant, is the same as for the ordinary open string field theory (OSFT). In that case we know however, that we should look for the solution of the full equations of motion outside the family of surface states.

The overlap equations encode all the information about the string field theory vertices. It would be interesting to see if we could derive more identities based on overlap equations for products of local operators. Such identities could then uniquely specify all the coefficients in the solution $[2,3]$, except for a single parameter.

In a recent paper [28] by Gaiotto et al. the authors found an intriguing coincidence for the OSFT solutions. The coefficients in the matter sector agree within few percent with coefficients of a solution of a ghost number zero string field theory, whose equation of motion in the Siegel gauge reads

$$
\left(L_{0}-1\right)|\Psi\rangle=-|\Psi\rangle *|\Psi\rangle
$$

Even though the solution is given purely in terms of total Virasoro operators acting on the vacuum, we can still find our quadratic identities based on $K_{n}$ 's in the matter sector. They happen to be the same as the matter part of such identities in the OSFT. Whether one can disentangle the matter and ghost sectors and add more identities to fully prove the observed pattern remains to be seen.

Another issue discussed in the paper are the surface states and projectors. We have shown that $K_{n}$ star algebra derivatives can be useful to calculate some nontrivial star products and to find some projectors. It would be interesting to obtain full classification 
of all projectors and to clarify their relation to star algebra derivatives in general.

\section{Acknowledgements}

I would like to thank L. Rastelli, A. Sen, W. Taylor and B. Zwiebach for useful discus-

sions. This work has been supported in part by DOE contract \#DE-FC02-94ER40818. 


\section{A $S L(2, \mathbb{R})$ group identities}

Let us take an $\operatorname{sl}(2, \mathbb{R})$ algebra with a central extension satisfying the commutation relations

$$
\begin{aligned}
{\left[T_{1}, T_{-1}\right] } & =2 T_{0}+a, \\
{\left[T_{0}, T_{1}\right] } & =-T_{1}, \\
{\left[T_{0}, T_{-1}\right] } & =T_{-1} .
\end{aligned}
$$

Then we have

$$
\begin{aligned}
e^{\alpha\left(T_{1}-T_{-1}\right)} & =e^{-\tanh \alpha T_{-1}}(\cosh \alpha)^{-\left(2 T_{0}+a\right)} e^{\tanh \alpha T_{1}} \\
e^{\alpha\left(T_{1}+T_{-1}\right)} & =e^{\tan \alpha T_{-1}}(\cos \alpha)^{-\left(2 T_{0}+a\right)} e^{\tan \alpha T_{1}} \\
e^{\alpha T_{1}} e^{\beta T_{-1}} & =e^{\frac{\beta}{1-\alpha \beta} T_{-1}}(1-\alpha \beta)^{-\left(2 T_{0}+a\right)} e^{\frac{\alpha}{1-\alpha \beta} T_{1}}, \\
e^{\alpha T_{1}} e^{\beta T_{0}} & =e^{\beta T_{0}} e^{\alpha e^{\beta} T_{1}}
\end{aligned}
$$

These identities can be presumably established by a variety of means. We have found them by constructing explicitly an infinite dimensional representation built by successive actions of $T_{-1}$ on some vacuum state $|0\rangle$ which is annihilated by $T_{0}$ and $T_{1}$ and then using the method of differential equations described in [14]. Much simpler way to obtain them is by analytic continuation from analogous equations for $S U(2)$ group.

When applied to subalgebras of the Virasoro algebra through the identification

$$
T_{1}=\frac{1}{n} L_{n}, \quad T_{-1}=\frac{1}{n} L_{-n}, \quad T_{0}=\frac{1}{n} L_{0}
$$

and

$$
a=\frac{c}{12} \frac{n^{2}-1}{n}
$$

we find easily the following important relations

$$
\begin{aligned}
e^{\alpha K_{1}} & =e^{\tan \alpha L_{-1}}(\cos \alpha)^{-2 L_{0}} e^{\tan \alpha L_{1}}, \\
e^{\alpha K_{2}} & =e^{-\frac{1}{2} \tanh 2 \alpha L_{-2}}(\cosh 2 \alpha)^{-L_{0}-\frac{c}{8}} e^{\frac{1}{2} \tanh 2 \alpha L_{2}}, \\
e^{\alpha K_{2 k}} & =e^{-\frac{1}{2 k} \tanh 2 k \alpha L_{-2 k}}(\cosh 2 k \alpha)^{-\frac{1}{k} L_{0}-\frac{c}{24} \frac{4 k^{2}-1}{k}} e^{\frac{1}{2 k} \tanh 2 k \alpha L_{2 k}},
\end{aligned}
$$

where $K_{n}=L_{n}-(-1)^{n} L_{-n}$ as usual. 


\section{B Transformation properties of the butterfly}

The regularized version of the simplest butterfly state $e^{s L_{-2}}|0\rangle$ is also a surface state, since as we know from [23]

$$
e^{s L_{2}}=U \frac{z}{\sqrt{1-2 s z^{2}}} .
$$

By straightforward use of Virasoro commutation relations or by the general techniques of [18] we may find various conservation laws for this state

$$
\begin{aligned}
\left(L_{0}-2 s L_{-2}\right) e^{s L_{-2}}|0\rangle & =0 \\
\left(L_{2}-(2 s)^{2} L_{-2}\right) e^{s L_{-2}}|0\rangle & =\frac{c s}{2} e^{s L_{-2}}|0\rangle, \\
\left(L_{2 k}-(2 s)^{k+1} L_{-2}\right) e^{s L_{-2}}|0\rangle & =\frac{c(k+1)}{8}(2 s)^{k} e^{s L_{-2}}|0\rangle, \quad k \geq 1 .
\end{aligned}
$$

We keep the central charge $c$ arbitrary. Applying simple method of differential equations (see e.g. [14]) one can derive from these conservation laws formulas for the action of finite conformal transformation

$$
\begin{aligned}
e^{v L_{0}} e^{s L_{-2}}|0\rangle & =e^{s e^{2 v} L_{-2}}|0\rangle \\
e^{v L_{2}} e^{s L_{-2}}|0\rangle & =(1-4 v s)^{-\frac{c}{8}} \exp \left(\frac{s}{1-4 v s} L_{-2}\right)|0\rangle \\
e^{v L_{2 k}} e^{s L_{-2}}|0\rangle & =\left(1-2 k v(2 s)^{k}\right)^{-\frac{c}{16} \frac{k+1}{k}} \exp \left(\frac{s}{\sqrt[k]{1-2 k v(2 s)^{k}}} L_{-2}\right)|0\rangle .
\end{aligned}
$$

By the same method, or using the formula (134) from Appendix A as a shortcut, one can derive the action of a finite reparametrization generated by $K_{2}=L_{2}-L_{-2}$

$$
e^{\alpha K_{2}} e^{s L_{-2}}|0\rangle=(\cosh 2 \alpha-2 s \sinh 2 \alpha)^{-\frac{c}{8}} \exp \left(\frac{2 s \cosh 2 \alpha-\sinh 2 \alpha}{2 \cosh 2 \alpha-4 s \sinh 2 \alpha} L_{-2}\right)|0\rangle .
$$

Note, that from this formula follows for $c=0$ and $s \in\left[-\frac{1}{2}, \frac{1}{2}\right)$

$$
\lim _{\alpha \rightarrow \infty} e^{\alpha K_{2}} e^{s L_{-2}}|0\rangle=e^{-\frac{1}{2} L_{-2}}|0\rangle .
$$

This is quite analogous to (44), in that infinite reparametrization of some set of states yields the butterfly projector. It can be proven along the same lines since the surfaces corresponding to $\langle 0| e^{\frac{1}{2} L_{2}}$ and $\langle 0| e^{s L_{2}}$ for $s \in\left[-\frac{1}{2}, \frac{1}{2}\right)$ can be glued together without gluing singularity to singularity. 


\section{Some formulas for the regulated GRSZ solution}

Here we collect some useful formulas for the regularized butterfly states in a twisted conformal field theory

$$
\left|\Psi^{s, \tilde{s}}\right\rangle=e^{s L_{-2}^{X}+\tilde{s} L_{-2}^{\prime}{ }^{g h}} c_{1}|0\rangle .
$$

Using (137) and (138), one can easily establish for this state (omitting the superscripts)

$$
\begin{aligned}
\left\langle\Psi\left|c_{0}\right| \Psi\right\rangle & =\left(1-4 s^{2}\right)^{-\frac{26}{8}}\left(1-4 \tilde{s}^{2}\right)^{\frac{2}{8}}, \\
\left\langle\Psi\left|c_{0} L_{0}^{X}\right| \Psi\right\rangle & =\frac{26 s^{2}}{1-4 s^{2}}\left\langle\Psi\left|c_{0}\right| \Psi\right\rangle, \\
\left\langle\Psi\left|c_{0} L_{0}^{\prime g h}\right| \Psi\right\rangle & =-\frac{2 \tilde{s}^{2}}{1-4 \tilde{s}^{2}}\left\langle\Psi\left|c_{0}\right| \Psi\right\rangle, \\
\left\langle\Psi\left|c_{0} L_{0}^{t o t}\right| \Psi\right\rangle & =\left(\frac{26 s^{2}}{1-4 s^{2}}-\frac{2 \tilde{s}^{2}}{1-4 \tilde{s}^{2}}-1\right)\left\langle\Psi\left|c_{0}\right| \Psi\right\rangle, \\
\left\langle\Psi\left|c_{0} L_{2 k}^{X}\right| \Psi\right\rangle & =26(2 s)^{k}\left(\frac{s^{2}}{1-4 s^{2}}+\frac{k+1}{8}\right)\left\langle\Psi\left|c_{0}\right| \Psi\right\rangle, \\
\left\langle\Psi\left|c_{0} L_{2 k}^{\prime g h}\right| \Psi\right\rangle & =-2(2 \tilde{s})^{k}\left(\frac{\tilde{s}^{2}}{1-4 \tilde{s}^{2}}+\frac{k+1}{8}\right)\left\langle\Psi\left|c_{0}\right| \Psi\right\rangle .
\end{aligned}
$$

\section{Multiplication of butterflies in level expansion}

In this appendix we present some numerical data showing how well the butterfly projectors behave in level expansion.

We have calculated first three coefficients of the product $e^{-\frac{1}{2} L_{-2}}|0\rangle * e^{-\frac{1}{2} L_{-2}}|0\rangle$ up to level 16. Calculation up to level $L$ means, that we truncate the input state up to that level and then perform the star multiplication exactly. The results are

\begin{tabular}{|c|c|c|c|}
\hline Level & $L_{-2}|0\rangle$ & $L_{-2} L_{-2}|0\rangle$ & $L_{-4}|0\rangle$ \\
\hline 2 & -0.4323 & 0.0937 & -0.04069 \\
\hline 4 & -0.4603 & 0.1084 & -0.00351 \\
\hline 6 & -0.4721 & 0.1131 & -0.00213 \\
\hline 8 & -0.4786 & 0.1157 & -0.00146 \\
\hline 10 & -0.4826 & 0.1173 & -0.00108 \\
\hline 12 & -0.4854 & 0.1185 & -0.00084 \\
\hline 14 & -0.4874 & 0.1193 & -0.00067 \\
\hline 16 & -0.4889 & 0.1200 & -0.00055 \\
\hline$\infty$ & -0.4955 & 0.1234 & -0.00019 \\
\hline exact & -0.5000 & 0.1250 & 0 \\
\hline
\end{tabular}


For the product $e^{\frac{1}{4} L_{-4}}|0\rangle * e^{\frac{1}{4} L_{-4}}|0\rangle$ we have calculated the first three coefficients up to level 24:

\begin{tabular}{|c|c|c|c|}
\hline Level & $L_{-2}|0\rangle$ & $L_{-2} L_{-2}|0\rangle$ & $L_{-4}|0\rangle$ \\
\hline 4 & -0.0709 & -0.00296 & 0.2418 \\
\hline 8 & -0.0494 & -0.00136 & 0.2452 \\
\hline 12 & -0.0398 & -0.00080 & 0.2466 \\
\hline 16 & -0.0342 & -0.00054 & 0.2473 \\
\hline 20 & -0.0304 & -0.00039 & 0.2478 \\
\hline 24 & -0.0276 & -0.00030 & 0.2482 \\
\hline$\infty$ & -0.0211 & 0.00025 & 0.2492 \\
\hline exact & 0 & 0 & 0.2500 \\
\hline
\end{tabular}

The values for level $L=\infty$ were obtained in both cases by a simple fit $a+\frac{b}{L}$. For some coefficients a different power of $L$ seems more accurate, but due to lack of data we refrain from more careful analysis. Anyway, the numerical results are compatible with butterflies being projectors.

Finally as a check on our methods and formulas we present numerical calculation of the star product (58)

$$
\begin{aligned}
e^{\frac{1}{2} L_{-2}}|0\rangle * e^{\frac{1}{2} L_{-2}}|0\rangle= & e^{\frac{1}{3} L_{-2}+\frac{5}{54} L_{-4}-\frac{5}{162} L_{-6}+\frac{59}{2916} L_{-8}+\cdots}|0\rangle \\
= & \left(1+0.3333 L_{-2}+0.0926 L_{-4}-0.0309 L_{-6}+0.0555 L_{-2} L_{-2}+\right. \\
& \left.\quad+0.0154\left(L_{-2} L_{-4}+L_{-4} L_{-2}\right)+0.00617 L_{-2} L_{-2} L_{-2}+\cdots\right)|0\rangle .
\end{aligned}
$$

Numerical calculation at level 16 gives

$$
\begin{aligned}
e^{\frac{1}{2} L_{-2}}|0\rangle * e^{\frac{1}{2} L_{-2}}|0\rangle= & \left(1+0.3001 L_{-2}+0.0928 L_{-4}-0.0272 L_{-6}+0.0452 L_{-2} L_{-2}+\right. \\
& +0.0140\left(L_{-2} L_{-4}+L_{-4} L_{-2}\right)+0.00388 L_{-2} L_{-2} L_{-2}+ \\
& \left.-0.00022 L_{-3} L_{-3}+\cdots\right)|0\rangle
\end{aligned}
$$

which is at least roughly compatible with the exact answer. 


\section{References}

[1] E. Witten, "Noncommutative Geometry And String Field Theory," Nucl. Phys. B268, 253 (1986).

[2] A. Sen and B. Zwiebach, "Tachyon condensation in string field theory," JHEP 0003 (2000) 002 [arXiv:hep-th/9912249].

[3] N. Moeller and W. Taylor, "Level truncation and the tachyon in open bosonic string field theory," Nucl. Phys. B 583 (2000) 105 [arXiv:hep-th/0002237].

[4] A. Sen, "Universality of the tachyon potential," JHEP 9912 (1999) 027 [arXiv:hepth/9911116].

[5] L. Rastelli, A. Sen and B. Zwiebach, "String field theory around the tachyon vacuum," arXiv:hep-th/0012251.

[6] L. Rastelli, A. Sen and B. Zwiebach, "Vacuum string field theory," hepth/0106010.

[7] D. Gaiotto, L. Rastelli, A. Sen and B. Zwiebach, "Ghost structure and closed strings in vacuum string field theory," arXiv:hep-th/0111129.

[8] H. Hata and T. Kawano, "Open string states around a classical solution in vacuum string field theory," JHEP 0111 (2001) 038 [arXiv:hep-th/0108150].

[9] K. Okuyama, "Ghost kinetic operator of vacuum string field theory," JHEP 0201 (2002) 027 [arXiv:hep-th/0201015].

[10] L. Rastelli, A. Sen and B. Zwiebach, "Boundary CFT construction of D-branes in vacuum string field theory," JHEP 0111 (2001) 045 [arXiv:hep-th/0105168].

[11] K. Okuyama, "Ratio of tensions from vacuum string field theory," arXiv:hepth/0201136.

[12] A. Sen, "Ghosts and vacuum string field theory," talk at JHS/60, Caltech 2001, http://quark.theory.caltech.edu/jhs60/program.html 
[13] M. Schnabl, "Constraints on the tachyon condensate from anomalous symmetries," Phys. Lett. B 504 (2001) 61 [arXiv:hep-th/0011238].

[14] M. Schnabl, "Wedge states in string field theory," arXiv:hep-th/0201095.

[15] D. J. Gross and A. Jevicki, "Operator Formulation Of Interacting String Field Theory I, II," Nucl. Phys. B283, 1 (1987);Nucl. Phys. B287, 225 (1987).

[16] S. Samuel, "The Physical and Ghost Vertices In Witten's String Field Theory," Phys. Lett. B181, 255 (1986).

[17] L. J. Romans, "Operator Approach To Purely Cubic String Field Theory," Nucl. Phys. B 298 (1988) 369.

[18] L. Rastelli and B. Zwiebach, "Tachyon potentials, star products and universality," JHEP 0109 (2001) 038 [arXiv:hep-th/0006240].

[19] B. Zwiebach, "Trimming the tachyon string field with SU(1,1)," arXiv:hepth/0010190.

[20] L. Rastelli, A. Sen and B. Zwiebach, private communication.

[21] L. Rastelli, A. Sen and B. Zwiebach, "Star algebra spectroscopy," arXiv:hepth/0111281.

[22] A. LeClair, M. E. Peskin and C. R. Preitschopf, "String Field Theory On The Conformal Plane. 1. Kinematical Principles," Nucl. Phys. B 317 (1989) 411.

[23] A. LeClair, M. E. Peskin and C. R. Preitschopf, "String Field Theory On The Conformal Plane. 2. Generalized Gluing," Nucl. Phys. B 317 (1989) 464.

[24] I. Ellwood, B. Feng, Y. He and N. Moeller, "The identity string field and the tachyon vacuum," JHEP 0107 (2001) 016 [hep-th/0105024].

[25] A. S. Schwarz and A. Sen, "Gluing Theorem, Star Product And Integration In Open String Field Theory In Arbitrary Background Fields," Int. J. Mod. Phys. A 6 (1991) 5387. 
[26] G. T. Horowitz and S. P. Martin, "Conformal Field Theory And The Symmetries Of String Field Theory," Nucl. Phys. B 296 (1988) 220.

[27] L. Bonora, D. Mamone and M. Salizzoni, "B field and squeezed states in vacuum string field theory," arXiv:hep-th/0201060.

[28] D. Gaiotto, L. Rastelli, A. Sen and B. Zwiebach, "Patterns in open string field theory solutions," arXiv:hep-th/0201159.

[29] H. Hata, S. Moriyama and S. Teraguchi, "Exact results on twist anomaly," arXiv:hep-th/0201177.

[30] I. Y. Aref'eva, D. M. Belov and A. A. Giryavets, "Construction of the vacuum string field theory on a non-BPS brane," arXiv:hep-th/0201197.

[31] J. Klusoň, "Exact Solutions of Open Bosonic String Field Theory," arXiv:hepth/0202045.

[32] M. R. Douglas, H. Liu, G. Moore and B. Zwiebach, "Open String Star as a Continuous Moyal Product," arXiv:hep-th/0202087.

[33] D. Gaiotto, L. Rastelli, A. Sen and B. Zwiebach, to appear. 